\title{
Status of KM3NeT
}

\section{P.Kooijman ${ }^{1}$ (for the KM3Net Consortium)}

University of Amsterdam-GRAPPA, University of Utrecht, Nikhef

Science Park 105, 1098 XG Amsterdam

E-mail: h84@nikhef.nl

The technical design report of the KM3NeT neutrino detector has been published. In this paper we describe the main features of this future telescope. The detector will be located on the floor of the Mediterranean Sea and will have a total volume of 6 to $8 \mathrm{~km}^{3}$. The point source sensitivity for sources near the Galactic Centre will be more than two orders of magnitude better than any other telescope. The technical solutions and innovations employed to achieve the quoted sensitivity will be presented.

35th International Conference of High Energy Physics (ICHEP2010)

Paris,France

July 22-28, 2010

\footnotetext{
$1 \quad$ Speaker
} 


\section{Introduction}

In the past four years a consortium of 40 institutes from 10 European countries has performed a design study ${ }^{1}$ for producing a cubic kilometre sized neutrino telescope in the Mediterranean Sea. The infrastructure will also host a deep-sea cabled infrastructure for marine sciences. The scope of the study was the design of an underwater neutrino telescope with a total volume of at least one cubic kilometre at a cost of less than M€250. The technical design report stemming from this design study has been published [1,2].

\section{Detection principle}

In a deep-sea neutrino telescope the neutrinos are detected by observing the Cherenkov radiation emitted by muons produced in charged current interactions of neutrinos with either the sea water or seabed in the vicinity of the telescope. The Cherenkov radiation is observed in a large array of optical sensors. These sensors are contained in glass vessels that can withstand the ambient hydrostatic pressure. The sensor in its vessel is called an optical module. The optical modules are suspended from vertical structures that are several hundred metres high. By placing many of these structures (detection units) over a large area of the sea bed a three dimensional array of sensors is produced. The direction and position of a traversing muon are reconstructed from the time and position correlations of photon hits in the optical modules. Timing accuracy of the order of a nanosecond and position accuracy of the order of a few tens of centimetres are required. The Mediterranean Sea offers an ideal environment for such a telescope. The absorption length of light in the water is very long at around $50 \mathrm{~m}$ allowing for a sparse array of sensors, whereas the scattering length is long so that over large distances the timing information does not deteriorate.

The present design study builds on the experiences of three pilot projects NEMO [3], NESTOR [4] and especially Antares [5]. Antares has now built up several years of experience of running a deep-sea telescope and has proved that the required accuracy in timing and positioning is attainable.

\section{Designs}

The main characteristic in determining the sensitivity of a neutrino telescope is the total area of photocathode per unit volume of water. During the design study two diverse methods of distributing this photocathode area over the volume have been investigated based on somewhat different philosophies (see Figure 1).

The "string" design [6] concentrates as much photocathode area as possible inside a single pressure vessel. This is achieved by the use of many (31) small (3 inch) phototubes inside a 17 inch glass pressure resistant sphere (DOM). Up to now the traditional way has been to house only one large (8 or 10 inch) tube inside such a sphere. The design with the small phototubes packs the equivalent photocathode area of more than 3 large tubes into a single sphere. All the necessary readout electronics is housed inside the sphere, creating a standalone sensor. This

\footnotetext{
${ }^{1}$ This design study was supported by European grant FP6: Contract number 011937 and FP7 under contract no. 212525
} 
method reduces the number of high pressure feed throughs to a minimum. Twenty such sensors are then suspended between two thin ropes separated at $30 \mathrm{~m}$ vertical intervals starting from 100 $\mathrm{m}$ from the seafloor. This then forms a vertical structure of $670 \mathrm{~m}$ height.
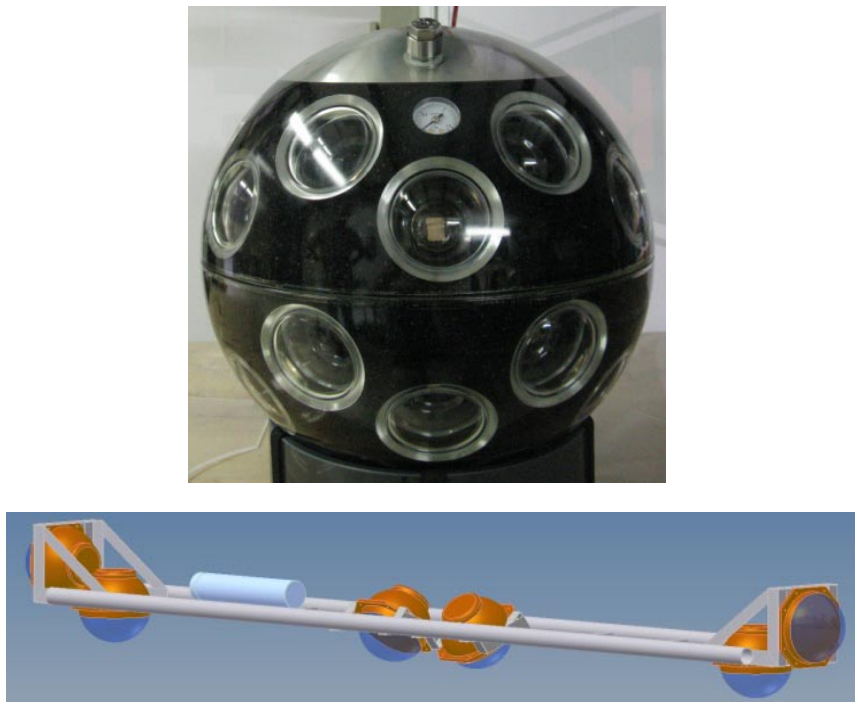
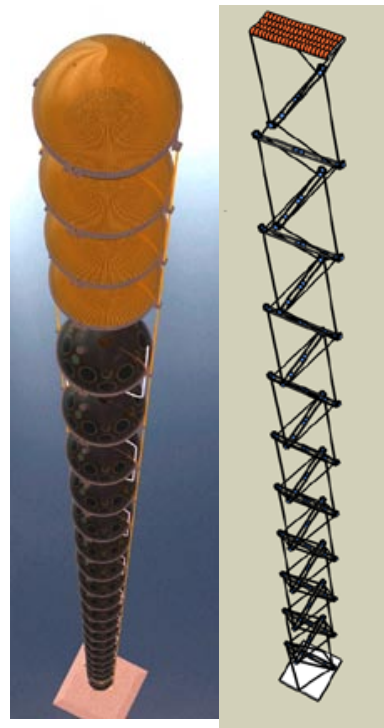

Figure 1: The DOM (top left), the $6 \mathrm{~m}$ long horizontal structure with six traditional optical modules (bottom left), the string detection unit (middle) and the tower detection unit (right). The vertical distances in the detection units has been reduced by a factor 10 .

The "tower" design [7] uses the traditional sensor with its readout electronics housed in a separate pressure resistant vessel. Six sensors and the electronics vessel are housed on $6 \mathrm{~m}$ long horizontal structures. Twenty of these are connected $40 \mathrm{~m}$ apart perpendicular to one another, to form a tower like structure (Figure 1). This structure houses twice as much photocathode area as the first design and has the advantage that the number of underwater structures and thereby the number of connections, that have to be made underwater, is reduced. The wet mateable connectors, capable of sustaining the pressures encountered at the 2500 to $4500 \mathrm{~m}$ anticipated as deployment depth, have proved in the past to be very expensive and lacking in reliability. At present an option of the horizontal structure housing two DOMs is being investigated. This would take advantage of the increased reliability of the DOM and the reduction of the underwater connections.

To readout the sensors a novel technique will be used [8]. It consists of a dense wavelength division multiplexing fibre optic system that connects each subsea electronics readout board to the shore via a single frequency channel. The system is driven by continuous wave lasers on shore, which pass up to 80 communications wavelengths down a single fibre to a junction box on the seabed. The single fibre is split into up to 160 fibres each carrying 40 wavelengths. Each of these fibres is fed to a master module in the detection unit, where the wavelengths are de-multiplexed. Each wavelength is sent via a bi-directional fibre to the optical module, where it is reflected and modulated using a reflective-electro-optical-amplifiermodulator (REAM). The modulated signal is multiplexed into 80 fibres each carrying the signals from 80 optical modules. A system is incorporated that can determine the time delay between REAM and shore to a measured accuracy of 50 ps over a distance of $100 \mathrm{~km}$. Finally 
the control signals from shore to the optical modules are broadcast via a modulation on the continuous wave laser. A schematic view of the system is given in Figure 2.

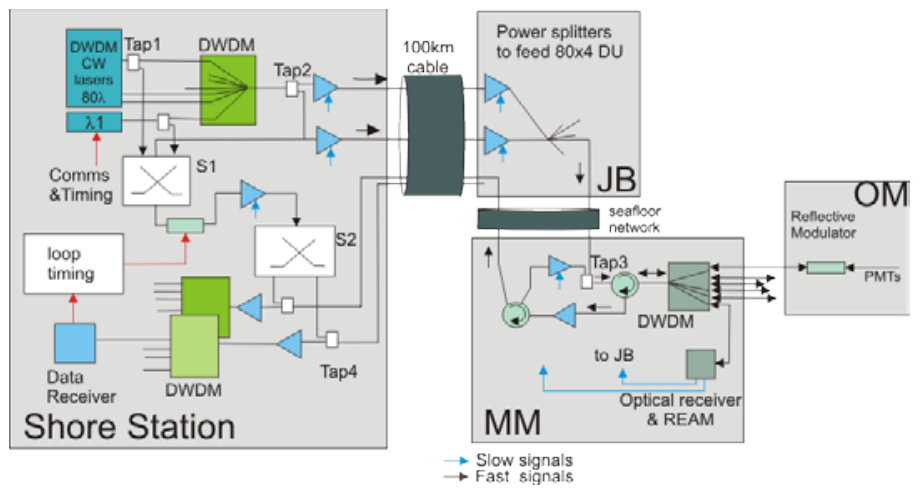

Figure 2: Schematic of the readout system

\section{Deployment tests}

Deployment tests of both detection unit structures have been performed. In both cases the detection units were deployed as a compact structure to the seafloor and released to unfurl. The string unit was rolled around a spherical launching unit that unrolls and releases the optical modules one by one. The tower unit is placed as a stack on the seafloor and the horizontal bars are raised one by one by a buoy (see Figure 3)
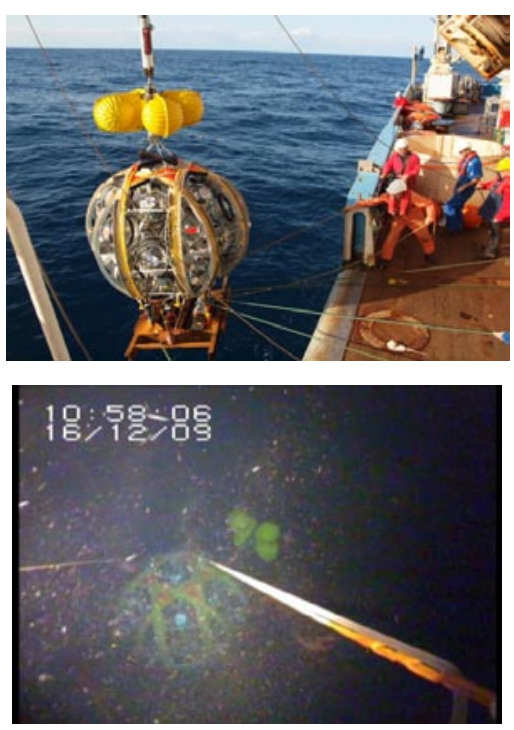
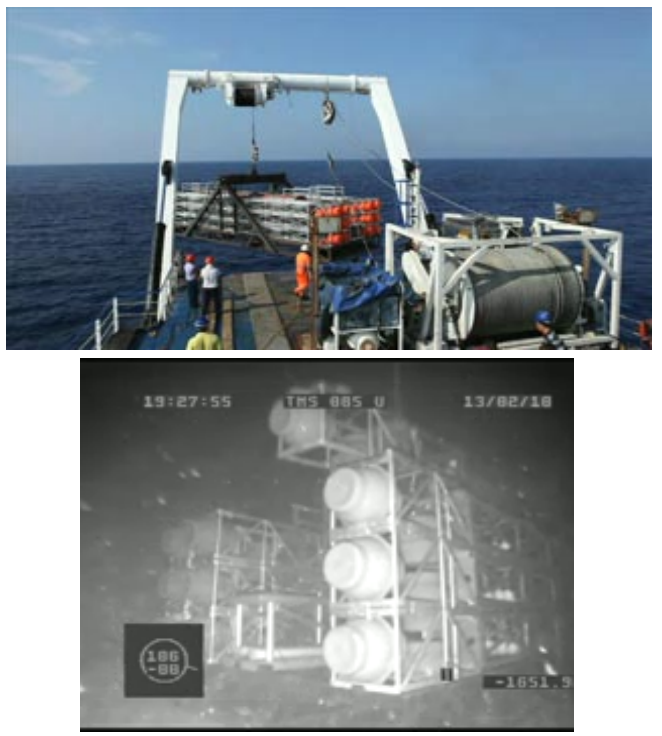

Figure 3: Deployment of the string on the spherical launcher (top left) and the release on from the seabed caught by the underwater camera (bottom left). The deployment of the tower unit in a stack (top right) and the release from the seafloor observed from the ROV submersible (bottom right).

\section{Performance}

The cost of the two designs have been calculated, and the number of tower (string) units that can be built for the foreseen budget is 320 (650). These can be placed at a distance of $180 \mathrm{~m}$ 
(130 m) apart, allowing for a detection volume of 6-8 $\mathrm{km}^{3}$ to be instrumented. The required human resources to build such a detector has been estimated at around 200-220 fte years, making a construction time of four to five years possible.

The sensitivity of these detectors has been determined by Monte Carlo simulation. The sensitivity for neutrino point sources with an $\mathrm{E}^{-2}$ energy spectrum is shown in Figure 4 together with the $5 \sigma$ discovery potential as a function of declination. This turns out to be independent of the chosen design. Also shown are the positions in declination of known high energy gamma ray sources. The sensitivities that can be obtained with the IceCube detector are also indicated. For sources near the centre of the Galaxy the sensitivity of KM3NeT is more than two orders of magnitude better than that of other detectors covering the same region in declination. Even at the point where IceCube has its maximum sensitivity KM3NeT performs better even though this region of the sky is only visible to KM3NeT $15 \%$ of the time.

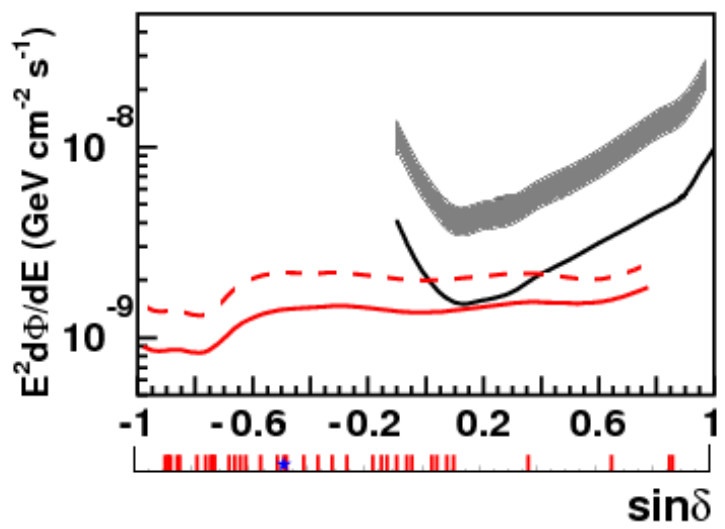

Figure 4: Sensitivity as a function of the sine of the declination angle. Red curves are for $3 \sigma$ exclusion (solid) and $5 \sigma$ discovery(dashed) for a one year exposure. The black curve and greay shaded area give the same for IceCube. The red dashes indicate the positions of known high energy gamma ray emitters.

\section{References}

[1] KM3NeT: Technical design report. ISBN 978-90-6488-033-9, 2010.

[2] KM3NeT. [Online]. http://www.km3net.org

[3] The NEMO Project. [Online]. http://nemoweb.lns.infn.it/project.htm

[4] The NESTOR neutrino telescope project.. [Online]. http://www.nestor.noa.gr/hena/intro/intro.htm

[5] The ANTARES neutrino telescope project. [Online]. http://antares.in2p3.fr/

[6] E. de Wolf, "A sensor architecture for neutrino telescopes," in Proc. of VLVnT, Athens, Greece, 2009.

[7] M. Musumeci, "A new design for the tower detection unit," in Proc. of VLVnT, Athens, Greece, 2009.

[8] J. Hogenbirk, "A photonic readout and data acquisition system for deep-sea neutrino telescopes," in Proc. of VLVnT, Athens, Greece, 2009. 